\title{
Memristör Temelli 2. Dereceden Aktif Yüksek Geçiren Filtrenin İncelenmesi
}

\author{
İshak PARLAR*, M. Nuri ALMALI \\ Van Yüzüncü Yıl Üniversite, Elektrik-Elektronik Müh. Bölümü, Van \\ (ORCID: 0000-0002-6344-4253) (ORCID:0000-0003-2763-4452)
}

\begin{abstract}
Öz
Bu çalışmada ilk olarak Alharbi, Muthuswamy ile Mutlu ve arkadaşları tarafindan literatürde önerilen memristör taklit devrelerinin Multisim programında düşük frekanslarda $(5 \mathrm{~Hz}-150 \mathrm{~Hz})$ benzetimi yapıldı. Taklit devreleri için farklı frekanslarda akım-gerilim (I-V) eğrileri incelenerek en kararlı çıkış eğrisini veren Mutlu ve arkadaşları tarafından önerilen devre gerçekleştirildi. Bu devre kullanılarak 2. dereceden aktif yüksek geçiren filtre devresi tasarlandı. Memristörlü ve direnç kullanılarak gerçekleștirilen filtre devreleri kalite faktörleri, Bode diyagramları ve kesim frekansları açısından karşılaş̧ıııldı. Memristörlü filtre devresinin 0-10 Hz frekans aralığında direnç kullanılarak gerçekleştirilen filtre devresinden daha iyi süzme işlemi yaptığı görüldü.
\end{abstract}

Anahtar kelimeler: Memristör, Filtre, Memristör Taklit Devresi.

\section{Investigation of Memristor Based Second Order Active High Pass Filter}

\begin{abstract}
In this study, firstly the emulator circuits proposed by Alharbi, Muthuswamy and Mutlu et al.were simulated low frequencies $(5 \mathrm{~Hz}-150 \mathrm{~Hz})$ in the Multisim program. For emulator circuits, current-voltage (I-V) curves of different frequencies were examined and circuit proposed by Mutlu and his friends who gave the most stable output curve was performed. By using this circuit, 2nd order active high pass filter circuit was designed. Filter circuits with built-in memristor and resistor; quality factors, Bode diagrams and cut frequencies were compared. It has been seen that the filter circuit with built-in memristor has been better filtered than resistor filter circuit which is used in the frequency range of $0-10 \mathrm{~Hz}$.
\end{abstract}

Keywords: Memristor, Filter, Memristor Emulator.

\section{Giriş}

İlk defa 1971 yılında Leon Chua tarafindan 4. pasif temel devre elemanı olan memristor teorik olarak ortaya konulmuştur. Ayrıca memristörü aynı zamanda manyetik akı ve yük arasında bir ilişkiye sahip nonlinear bir hafiza elemanı olarak da tanımlandı [1]. Memristör, pratikte Williams ve ekibi tarafindan 2008 yılında Hewlett-Packard (HP) laboratuvarlarında fiziksel olarak üretildi. Bundan sonra, memristör analog devre tasarımlarında yaygın bir şekilde kullanılmaya başlandı. Memristörün popülaritesi ise her geçen gün artmaya devam etmiştir [4,14].

Literatürde son yıllarda memristör tabanlı filtreler üzerine yoğun bir şekilde çalışmalar yapıldı̆̆ 1 görülmektedir. Memristör tabanlı filtrelerin davranışı ve üzerindeki etkileri araştırılmakta ve zamanfrekans özelliklerini incelemek için çalışmalar yürütülmektedir [2,3,5,6,10,11,13].

Yener ve ark. 2014 yılında yaptıkları çalışmada, yeni programlanabilir amplifikatörler, adaptif filtreler ve programlanabilir osilatörler, yeni bir temel devre elemanı memristör kullanılarak tasarlanabileceğini ortaya atmışlardır. Yaptıkları çalışmada doğrusal sürüklenme memristör modeli kullanılarak bir memristör tabanlı biquad analog filtresini incelemişlerdir. Simülasyonlarda $\mathrm{TiO}_{2}$ memristörün lineer

*Sorumlu yazar: ishakparlar@yyu.edu.tr

Geliş Tarihi: 29.05.2019, Kabul Tarihi: 31.07.2019 
dopant sürüklenme modelini kullandıkları görülmektedir. Simülasyonlarda kazanç ve kalite faktörü gibi filtre bileşenlerinin biquad filtresindeki memristör kullanarak ayarlanabileceğini gösterdiler [13].

Sözen ve Çam, $\mathrm{TiO}_{2}$ memristör modellini pasif $\mathrm{RC}$ yüksek geçiren filtre devresine uygulamışlardır. Bu çalışmada memristörün başlangıç direnci olan Rinit değerini belirli oranlarda arttırıldığında kesim frekansı üzerine olan etkileri incelenmiştir. Artan Rinit değerinin, doğrudan $\mathrm{R}_{\mathrm{M}}{ }^{\prime} \mathrm{yi}$ etkilediği için filtre devresinin kesme frekansını azalttığını gözlemlemişlerdir [11].

Kirilov ve ark., Williams'ın 2008 yılındaki yaptığı çalışmada, piyasaya sürdüğg̈ memristör modeli kullanılarak pasif band geçiren filtre devresini $100 \mathrm{~Hz}-10 \mathrm{kHz}$ frekans aralı̆̆ında test ederken band durduran filtre devresini ise $1 \mathrm{kHz}-100 \mathrm{kHz}$ frekans aralığında test ettiler. $20 \mathrm{~Hz}$ 'den daha yüksek frekanslarda, Williams'ın memristörü lineer bir direnç gibi benzer bir davranışa sahip olduğunu ve memristör devresinden geçen sinyallerdeki bozulmaların önemsiz olduğunu belirtti. Kullanılan memristör modelinin farklı memristans noktalarına göre analiz edilerek sistemin tepkisini ölçmeye çalıştılar. Çalışmanın sonucunda, filtrelerde kullanılan memristörlerin özelliklerinin çalışma frekansı aralığında doğrusal hale geldiğini gördüler [6].

Yapılan literatür araştırmalarında Kirilov ve ark., Yener ve ark., gibi ekiplerin belli bir taklit devresini baz aldıkları ve sınırlı bir frekans aralığında çalıştıkları görülmektedir. Bu çalışmalar birden fazla taklit devresini baz alarak en iyi süzme işleminin belirlendiği filtre seçilerek ve bu filtre üzerindeki etkileri incelenerek gerçekleştirildi.

Bu çalışmada ilk olarak; Alharbi, Mutlu ve arkadaşları tarafından önerilen taklit devrelerinin Multisim programında benzetimi yapıldı. Daha sonra histerezis eğrisi üzerinde alçak frekans tepkisine sahip model kullanılarak 2. dereceden aktif yüksek geçiren filtrenin kalite faktörü, Bode diyagramı, I-V tepkisi ve kesim frekansları karşılaştırıldı. Son olarak elde edilen bulgular paylaşıldı ve elde edilen sonuçlar verilerek tartışıldı.

\section{Materyal ve Metot}

\subsection{Taklit Devrelerinin Matematiksel Modelleri}

Chua, çok sayıda transistör, işlemsel yükselteç, direnç vb. devre elemanları ile simüle edilmiş bir devre geliştirmişti [4]. Bu devre pek çok elemandan oluştuğu ve uygulanması uzun zaman aldığı için daha az elemandan oluşan ve uygulaması daha pratik olan birçok devre modeli sunulmuştur. Bu çalışmada 3 farklı taklit devresi modeli sunulmuştur. Model-1, Mutlu ve arkadaşları tarafindan önerilen taklit devresini, Model-2 Muthuswamy tarafindan önerilen gerilim kontrollü taklit devresini, Model-3 ise Alharbi ve arkadaşları tarafindan önerilen bir başka gerilim kontrollü taklit devresini göstermektedir.

Mutlu ve arkadaşları tarafindan kullanılan $\mathrm{TiO}_{2}$ Memristör modeli için dışardan harici bir öngerilim gerilimi $\mathrm{v}(\mathrm{t})$ verilmesi, kutuplarını kutuplarına göre iki bölge arasında sınırlandırır. Memristor için gerilim-akım ilişkisi Eş.(1) ile verilmiştir [5].

$$
v(t)=R_{O N} \frac{w(t)}{D}+R_{O F F}\left(1-\frac{w(t)}{D}\right) i(t)
$$

$w(t)=\mu_{V} \frac{R_{O N}}{D} q(t), \mu_{V}$ ortalama kayma hızı ve $\mathrm{cm}^{2} / \mathrm{s} V$ birimine sahiptir, D katkılı ve katkısız alanların toplamı ve m birimine sahiptir ve $\mathrm{q}(\mathrm{t})$ memristor cihazından geçen toplam yüke karş1l1k gelmektedir.

Mutlu ve arkadaşları tarafından önerilen lineer sürüklenme hızlı $\mathrm{TiO}_{2}$ memristör taklit devresi şematiği Şekil 1'de gösterilmiştir [9]. 


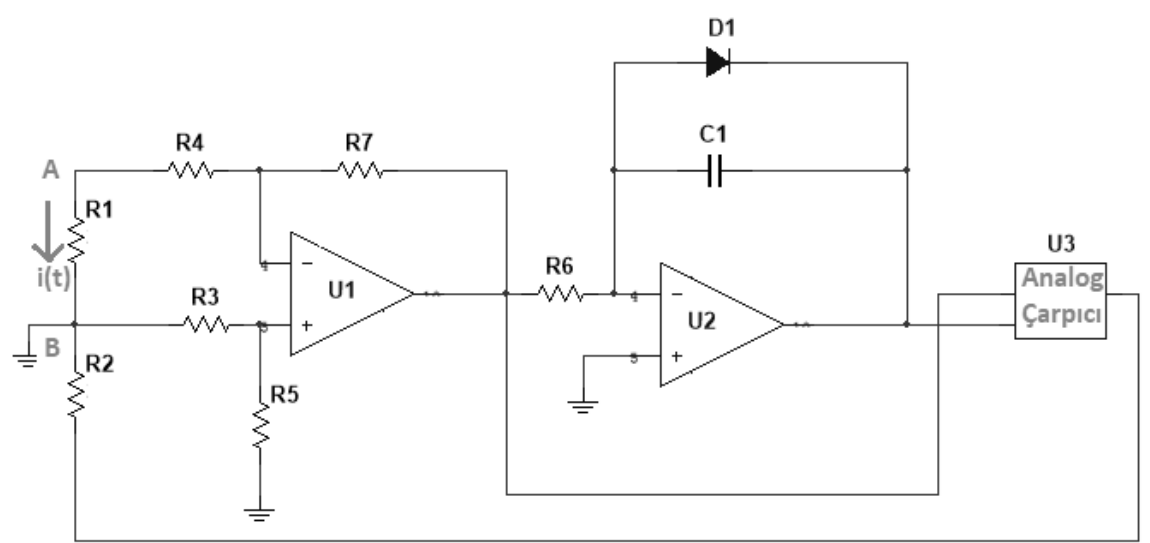

Şekil 1. Lineer Sürüklenme Hızlı $\mathrm{TiO}_{2}$ Memristör Taklit Devresi Şematiği [9]

Şekil 1'de verilen taklit devresi bir diferansiyel yükseltici, bir integral alıcı devresi ve bir analog çoklayıcıdan oluşmaktadır. Şekil 1'de verilen $i(t)$, A'dan B'ye akan akım olarak kabul edilirse devreden geçen yük $q(t)$, Eş. (2)'deki gibi ifade edilir.

$$
q(t)=\int_{t=-\infty}^{t} i(t) d t
$$

Memristör yükünün negatife gitmemesi için Şekil-1'de görülen diyot (D1) veya çok yüksek bir direnç değeri kullanılabilir. Aşağıdaki formülde, memristör yükü ve akımı birbiri çarpılmaktadır. Analog çarpıcıdaki gerilim ifadesi ise Eş. (3)'deki gibi gösterilmiştir.

$$
V_{C A R P I C I}=V_{U 3}=\left(\frac{-R_{3} R_{1}}{R_{2}}\right)^{2} \frac{1}{R_{6} C_{1}} q(t) i(t)
$$

Muthuswamy tarafindan önerilen devrenin matematiksel denklemleri Eş. (4)'te gösterilmiştir. Şekil 2'deki devre modelinde, AD711 işlemsel yükselteci ve AD633 çoklayıcı, integral alıcı ve diferansiyel amplifikatör devreleri için kullanılmıştır [8].

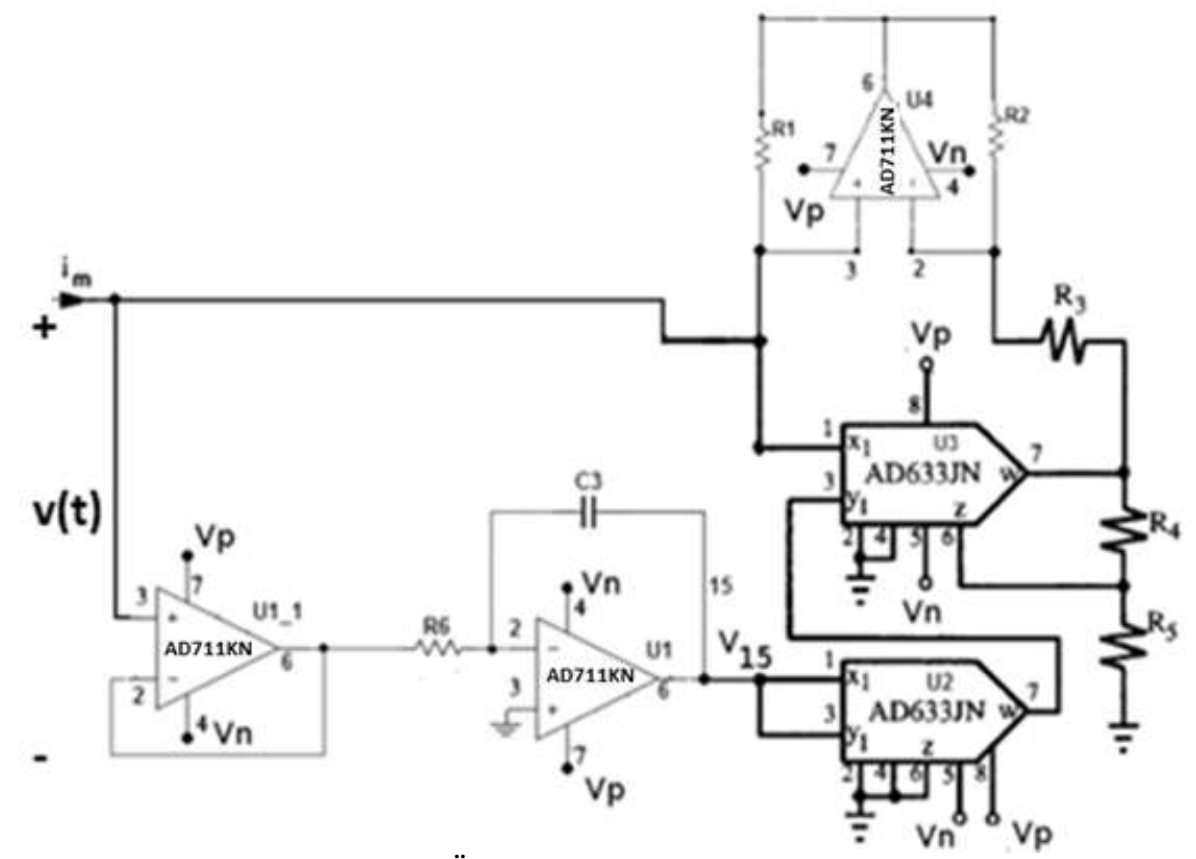

Şekil 2. Muthuswamy Tarafindan Önerilen Gerilim Kontrollü Memristör Taklit Devresi [8] 
Şekil 2'deki devre için kübik doğrusal olmayan $i_{m}(t)$ akımına ait ifade Eş.(4)'teki gibi yazılabilir [8]:

$$
i_{m}(t)=\left(\alpha+\beta 3\left(\int v(t) d t\right)^{2}\right) v(t)
$$

Burada memristör ölçüm değerlerine sahip $\alpha$ ve $\beta$ ifadeleri aşağıda gösterilmiştir.

$$
\alpha=-\frac{1}{R_{3}}, \quad \beta=\frac{1}{3}\left(\frac{R_{4}+R_{5}}{R_{3} \cdot R_{4} \cdot 100}\right)
$$

AD711 işlemsel yükselteci, akım invertörü olarak görev yapar ve eğer devrede $R_{1}=R_{2}$ ise Eş. (4), Eş. (5)'teki ifadeye dönüşür:

$$
i_{m}(t)=\frac{-v}{R_{3}}+\left(\frac{\int v(t) d t^{2} v\left(R_{4}+R_{5}\right)}{100 R_{4}}\right) \frac{1}{R_{3}}
$$

Alharbi ve ark., ise Şekil 3 'te verilen gerilim kontrollü bir memristör için genel ve basit bir taklit devresi modeli önerdiler [2].

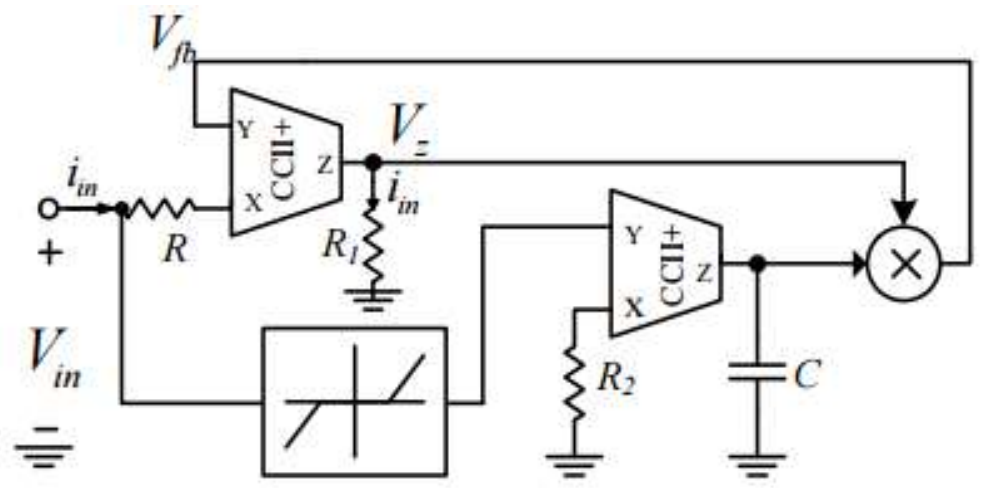

Şekil 3. Gerilim Kontrollü Memristör Taklit Devresi [2]

Şekil 3’te verilen taklit devresinde memristörün histerezis davranışını sağlaması için, iki adet ikinci nesil akım taşıyıcı (CCII+) ve analog çarpıcı kullanılmıştır. Devrenin giriş akımına ait ifade Eş. (6)'teki gibi yazılır [2]:

$$
i_{\text {in }}=\frac{V_{i n}-V_{f b}}{R}
$$

Burada $V_{f b}$ geri besleme gerilimini (çarpanın çıktısı) temsil etmektedir. Giriş gerilimi doğrusal olmayan bir fonksiyon $f\left(V_{i n}\right)$ kullanılarak şekillendirilmiştir. Çıkış voltajı ikinci CCII+, $V_{Z}$ gerilimi ile entegre edilir ve Eş. (7)'de olduğu gibi çarpılır ve geri besleme gerilimi üretilir:

$$
V_{f b}=\frac{\alpha V_{Z}}{R_{2} C} \int_{0}^{t} f\left(V_{i n}(t)\right) d t
$$

Burada $\alpha$ çarpım sabiti ve $\mathrm{f}$ ise şekillendirme fonksiyonudur. Giriş gerilimi ise Eş. (8)'deki gibi ifade edilmektedir.

$$
V_{\text {in }}=R+\frac{\alpha R_{1}}{R_{2} C} \int_{0}^{t} f\left(V_{\text {in }}(t)\right) d t i(t)
$$




\section{Bulgular ve Tartışma}

Bir önceki kısımda verilen üç farklı taklit devresi modelleri referans alınarak, bu devrelerin girişlerine ortak 2Vpp sinüs sinyali verilmiştir. Giriş sinyalinin frekans1, sırasıyla $5 \mathrm{~Hz}, 10 \mathrm{~Hz}, 15 \mathrm{~Hz}$ ve $150 \mathrm{~Hz}$ için akım-gerilim (histerezis eğrisi) eğrileri Şekil 4’te gösterilmiştir.

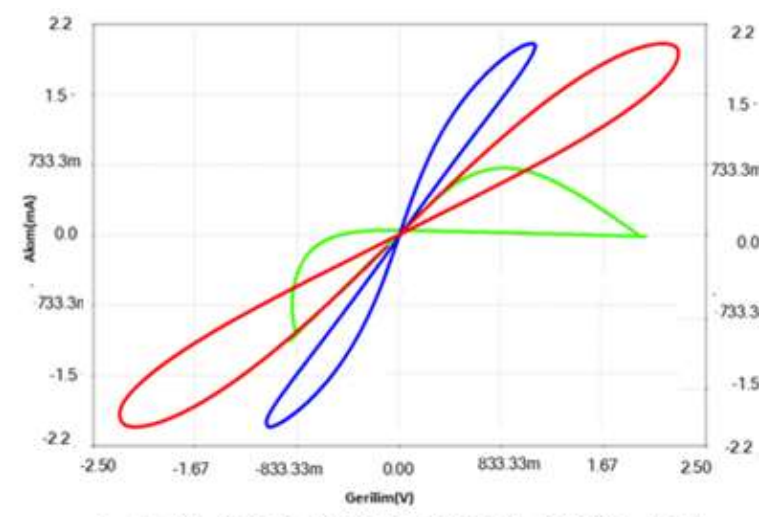

Modet -1 (R1=1K $\Omega, R 2=30 \mathrm{~K} \Omega, \mathrm{R} 3=470 \mathrm{~K} \Omega, \mathrm{R} 6=220 \mathrm{~K} \Omega, \mathrm{C}=10 \mathrm{nF}$ ) 8 Modet 2 (R3=1.69K $\Omega, R 4=30 \mathrm{~K} \Omega, \mathrm{R} 5=29 \mathrm{~K} \Omega, \mathrm{C}=100 \mathrm{nF}$ ) $\checkmark$ Model -3 ( $R=1 \mathrm{~K} \Omega, \mathrm{R} 1=5 \mathrm{~K} \Omega, \mathrm{R} 2=1 \mathrm{~K} \Omega, \mathrm{C}=100 \mathrm{uF})$

a)

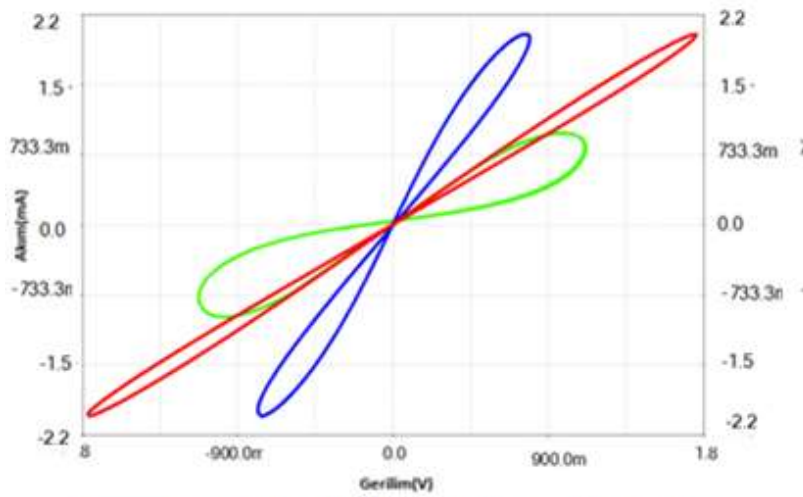

$\checkmark$ Model-1 (R1=1K $\Omega, R 2=30 \mathrm{~K} \Omega, \mathrm{R} 3=470 \mathrm{~K} \Omega, \mathrm{R} 6=220 \mathrm{~K} \Omega, \mathrm{C}=10 \mathrm{nF}$ ) ح. Model 2 ( $(R 3=1.69 \mathrm{~K} \Omega, \mathrm{R} 4=30 \mathrm{~K} \Omega, \mathrm{R} 5=29 \mathrm{~K} \Omega, \mathrm{C}=100 \mathrm{nF}$ ) $\checkmark$ Model 3 ( $R=1 \mathrm{~K} \Omega, \mathrm{R} 1=5 \mathrm{~K} \Omega, \mathrm{R} 2=1 \mathrm{~K} \Omega, \mathrm{C}=100 \mathrm{uF}$ )

c)

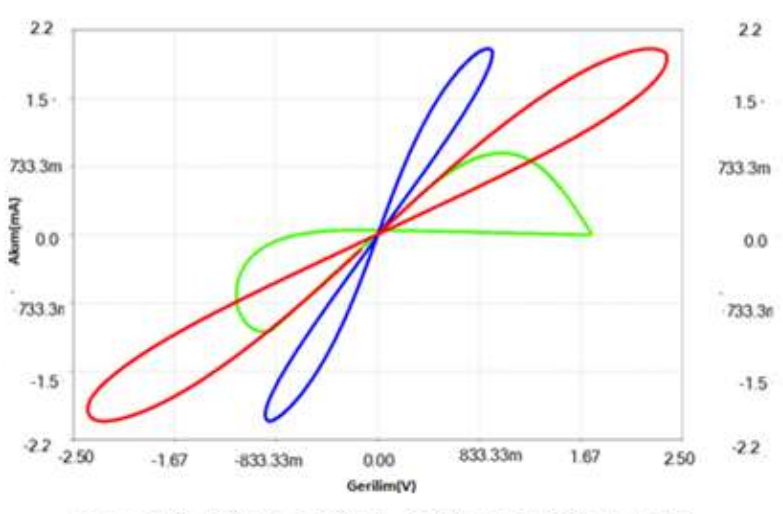

Modet -1 (R1=1K $\Omega, R 2=30 \mathrm{~K} \Omega, \mathrm{R} 3=470 \mathrm{~K} \Omega, \mathrm{R} 6=220 \mathrm{~K} \Omega, \mathrm{C}=10 \mathrm{nF}$ ) $\nabla$ Model 2 (R3=1.69K $\Omega, R 4=30 \mathrm{~K} \Omega, \mathrm{R} 5=29 \mathrm{~K} \Omega, \mathrm{C}=100 \mathrm{nF}$ ) Model 3 ( $R=1 \mathrm{~K} \Omega, R 1=5 \mathrm{~K} \Omega, \mathrm{R} 2=1 \mathrm{~K} \Omega, \mathrm{C}=100 \mathrm{uF})$

b)

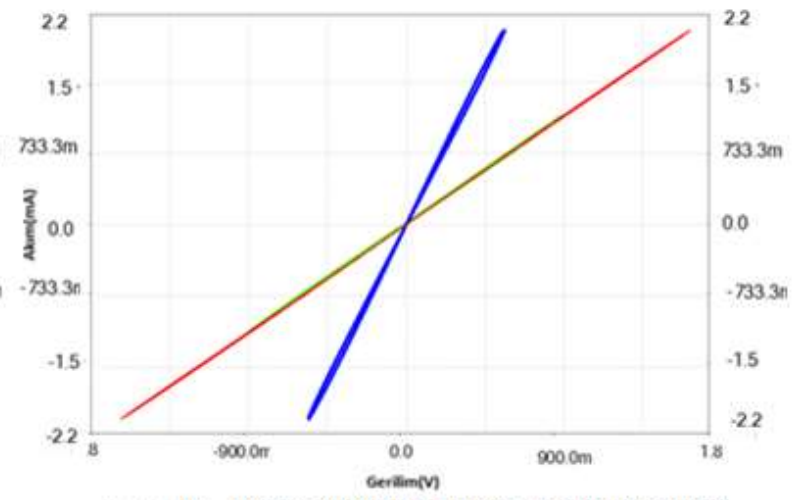

$\triangle$ Model-1 ( $R 1=1 \mathrm{~K} \Omega, \mathrm{R} 2=30 \mathrm{~K} \Omega, \mathrm{R} 3=470 \mathrm{~K} \Omega, \mathrm{R} 6=220 \mathrm{~K} \Omega, \mathrm{C}=10 \mathrm{nF}$ ) $\triangle$ Model 2 (R3=1.69k $\Omega, R 4=30 \mathrm{~K} \Omega, \mathrm{RS}=29 \mathrm{~K} \Omega, \mathrm{C}=100 \mathrm{nF}$ ) $\checkmark$ Modet 3 ( $R=1 \mathrm{~K} \Omega, R 1=5 \mathrm{~K} \Omega, \mathrm{R} 2=1 \mathrm{~K} \Omega, \mathrm{C}=100 \mathrm{FF}$ )

d)

Şekil 4. Taklit Devrelerinin Histerezis Eğrileri a) $2 \mathrm{Vpp}, 5 \mathrm{~Hz} \quad$ b) $2 \mathrm{Vpp}, 10 \mathrm{~Hz} \quad$ c) $2 \mathrm{Vpp}, 15 \mathrm{~Hz}$ d) $2 \mathrm{Vpp}, 150 \mathrm{~Hz}$

Tüm modeller öncelikle $2 \mathrm{Vpp}$ ve $5 \mathrm{~Hz}$ giriş sinüzoidal bir sinyal için benzetim çalışması yapılmıştır. $5 \mathrm{~Hz}$ frekansa sahip giriş sinyali için memristörün karakteristik eğrisi olan histerezis eğrisinin gerilimi sabit tutularak frekansı arttırdıkça giderek kaybolduğunu ve $150 \mathrm{~Hz}$ frekansa getirdiğimizde tamamen kaybolduğu gözlemlenmiştir. Yüksek frekanslarda memristive özelliğin kaybolduğu ancak bu karakteristiğin belirli frekans aralıklarında tekrarlandığı görülmüştür. Literatürde en sık kullanılan ve pratik uygulaması rahat bir şekilde gerçekleştirilen bu modeller ayrı ayrı incelenmiştir. Uygulama deneylerinde en kararlı çıkış tepkisini veren, $150 \mathrm{~Hz}$ frekansta histerezis alanın en az olduğu yani tamamen lineer özellik gösteren ve daha az aktif devre elemanı ile oluşturulan Model1 devresi ele alınmıştır.

Şekil 5'te gösterilen taklit devresi, Mutlu ve arkadaşları tarafindan önerilen lineer sürüklenme hızlı $\mathrm{TiO}_{2}$ memristör taklit devresinden (Model-1) yararlanılarak oluşturulmuştur [9,10]. 


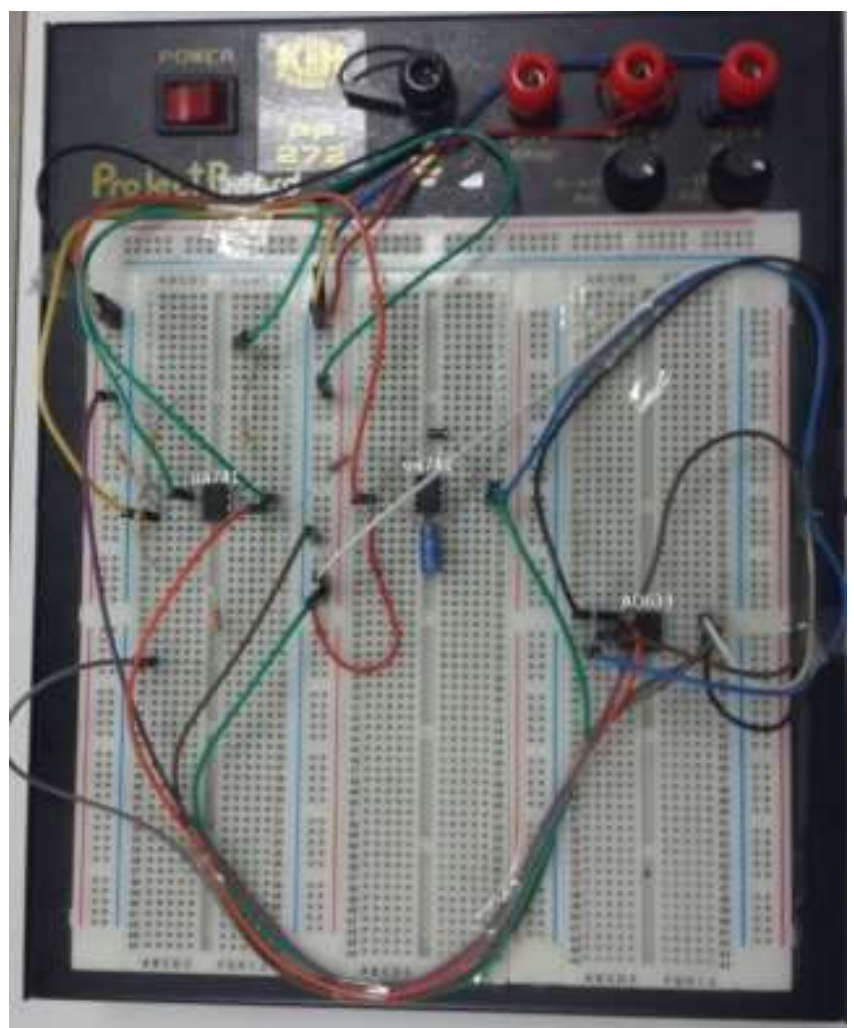

Şekil 5. Elektronik çalışma tahtası üzerine kurulmuş taklit devresi uygulaması

Öncelikle referans aldığımız Model-1 devresini elektronik çalışma tahtası üzerinde uygulama devresi tasarlanmıştır. Uygulamasını yaptığımız model-1 devresini analog devrelerde kullanmak için düşük frekanslarda histerezis alanının büyük olduğunu frekansı arttırdığımızda ise bu alanın azaldığını hatta lineer bir davranışa doğru gittiğini gözlemlememiz gerekmektedir. Akım ve gerilim parametreleri giriş sinyalinin frekansı arttırılarak tepkileri Şekil 6-7' de gösterilmiştir. 8Hz giriş sinyalinde akım lineer bir eğilim göstermediği ancak $150 \mathrm{~Hz}$ giriş sinyalinde lineer bir davranış sergilediği Şekil 10'da gözlemlenmiştir.

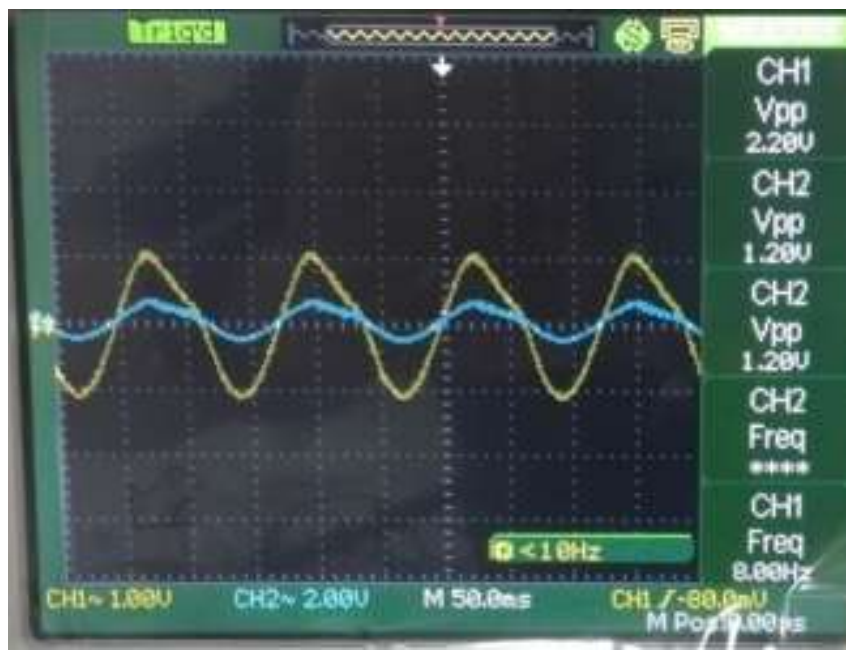

Şekil 6. $8 \mathrm{~Hz}$ Sinüsoidal gerilimle beslenen memristör taklitçisinin akım ve gerilim grafiği (CH1: Gerilim, CH2: Akım)

Giriş sinyal frekansı $150 \mathrm{~Hz}$ 'e ayarlanırsa, taklit devresi lineer bir direnç gibi davrandığı görülmüsstür. $150 \mathrm{~Hz}$ Sinüsoidal Gerilimle Beslenen Memristör Taklitçisinin Akım ve Gerilim Grafiği Şekil 7'de gösterilmiştir. Ayrıca çıkış akımı ve geriliminde distorsiyon (gürültü) meydana gelmemiştir. 


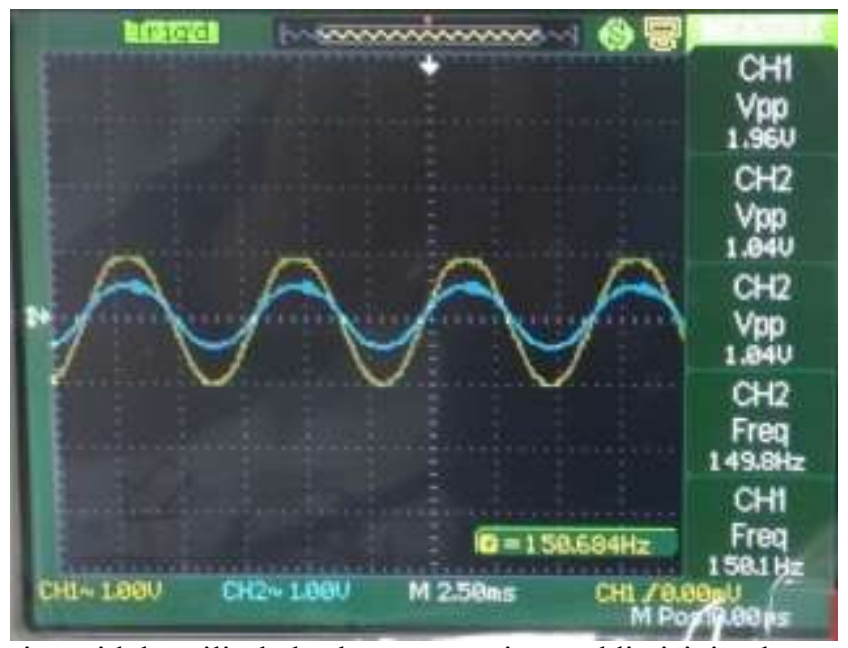

Şekil 7. $150 \mathrm{~Hz}$ sinüsoidal gerilimle beslenen memristör taklitçisinin akım ve gerilim grafiği (CH1: Gerilim, CH2: Akım)

Akım ve gerilim tepkileri verilen taklit devresini, X-Y düzleminde sırasıyla 8-9-150 Hz frekansa sahip sinüsoidal bir giriş sinyali uygulayarak histerezis eğrileri Şekil 8-9-10' da gösterilmiştir. $8 \mathrm{~Hz}$ frekansa sahip histerezis alanı (loop) net bir şekilde görülmektedir.

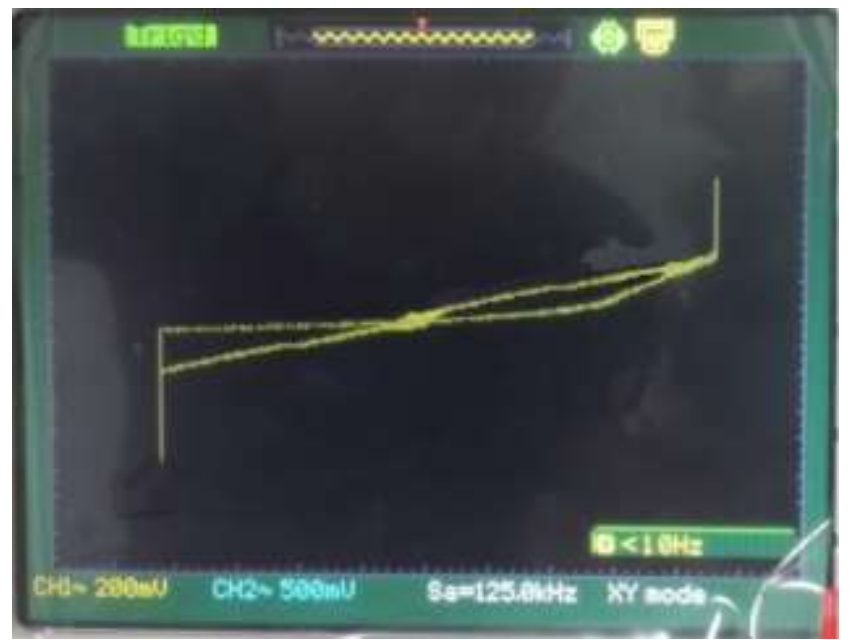

Şekil 8. $8 \mathrm{~Hz}$ sinüsoidal gerilimle beslenen memristör taklitçisinin histeresiz eğrisi

Giriş gerilimi değiştirilmeden frekansı 9 Hz'e yükselttiğimizde histerezis alanının azaldığı Şekil 9'da görülmektedir.

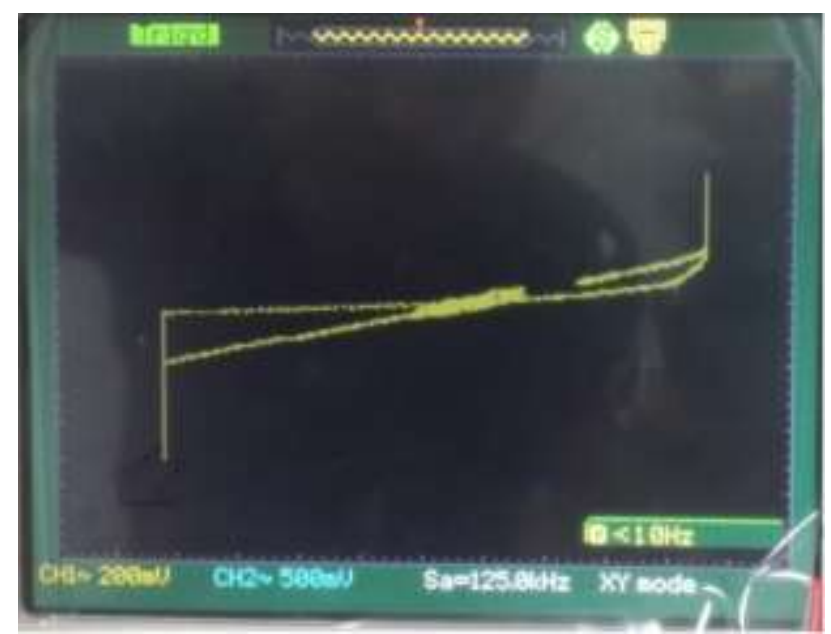

Şekil 9. $9 \mathrm{~Hz}$ sinüsoidal gerilimle beslenen memristör taklitçisinin histeresiz eğrisi 
Frekansı 150 Hz'e yükselttiğimizde ise histerezis alanının neredeyse kaybolduğu Şekil 10'da gözlemlenmiştir.

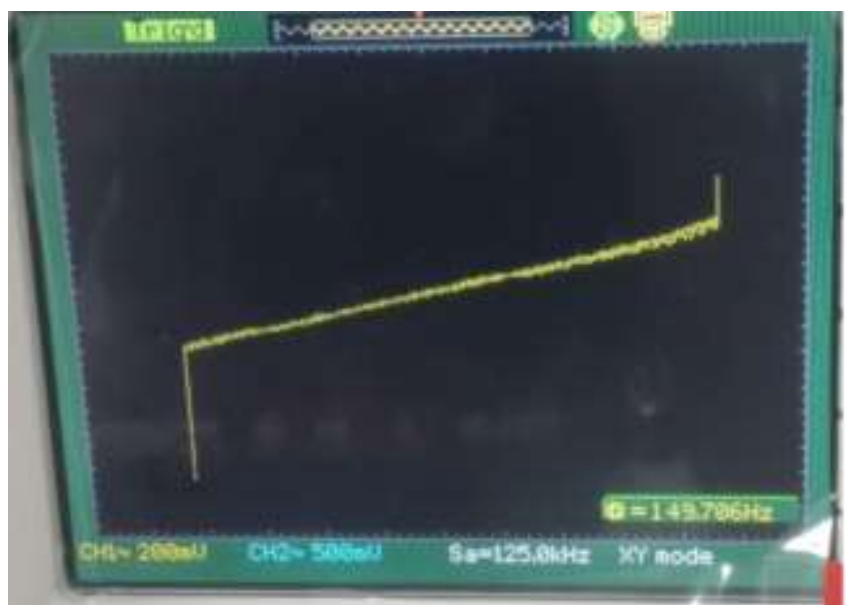

Şekil 10. $150 \mathrm{~Hz}$ sinüsoidal gerilimle beslenen memristör taklitçisinin histeresiz eğrisi

Aktif yüksek geçiren filtre (YGF), belli bir kesim frekansının altındaki sinyalleri zayıflatarak ve bu frekansın üzerindeki sinyalleri ise güçlendirerek çıkışa vermektedir. Kesim frekansının üzerindeki frekanslar için çıkış geriliminin genliği sabittir. İkinci dereceden yüksek geçiren filtrenin genel devresi Şekil 11'de gösterilmiştir [7].

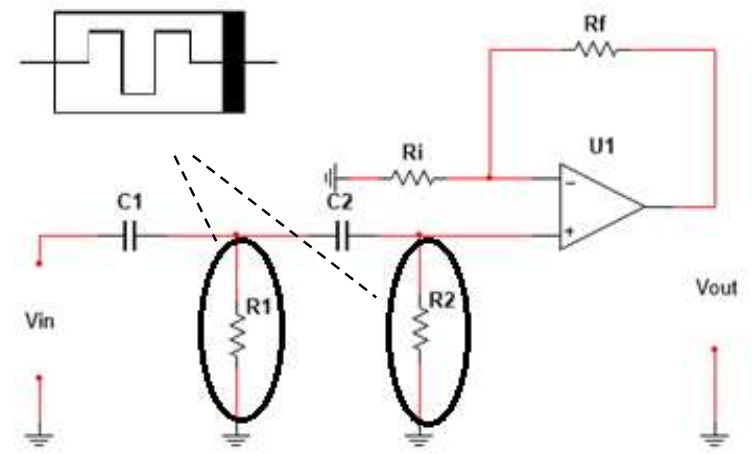

Şekil 11. İkinci dereceden geleneksel yüksek geçiren filtre devre şematiği [7]

Şekil 11'de verilen sistemin transfer fonksiyonu Eş. (9)'da verilmiştir. Bu denklemde $\mathrm{R}_{1}$ ve $\mathrm{R}_{2}$ dirençleri yerine eş olarak memristör taklit devreleri yerleştirilmiştir. $\left(R_{f}-R_{i}\right)$ dirençleri ise kazancı belirlemektedir. Uygulamada uygun $\mathrm{R}$ ve $\mathrm{C}$ değerleri seçilerek kesim frekansı olabildiğince düşük seçilmeye çalış1lmıştır. $\left(\mathrm{R}_{1}=\mathrm{R}_{2}=\mathrm{R}=900 \mathrm{~K} \Omega, \mathrm{C}_{1}=\mathrm{C}_{2}=\mathrm{C}=35 \mu \mathrm{F}\right)$

$$
\frac{V_{\text {out }}(S)}{V_{\text {in }}(S)}=\frac{s^{2} R_{4}\left(R_{i}+R_{f}\right) C^{2}}{s^{2} R_{M}{ }^{3} R_{i} C^{2}+s 3 R_{M}{ }^{2} R_{i} C+R_{M} R_{i}}
$$

$\mathrm{R}_{\mathrm{M}}$, genlik memristorünün direncini belirtir. Talukdar ve arkadaşları, giriş gerilimi olarak sinüzoidal bir dalga formu verilmesi durumunda, $\mathrm{R}_{\mathrm{M}}, \mathrm{R}_{\mathrm{avg}}$ boyunca salınacak ve Eş.(10)'daki gibi sinüzoidal giriş gerilimi için memristor direncini verecektir [12];

$$
R_{M}=R_{\text {avg }} \pm \Delta R_{M} \sin (w t+\varphi)
$$

Memristör direncin uç zirveleri ise Eş. (11) 'deki gibi verilebilir.

$$
R_{\text {max } \min }=R_{\text {avg }} \pm \Delta R_{M}
$$


Şekil 11'de verilen devrenin kesim frekansı ve kalite faktörü denklemleri sırasıyla Eş. (12-13)'te verilmiştir. Kalite faktörü $(Q)$, sistemin kesim frekansının band genişliğine $(B W)$ olan oranıdır.

$$
\begin{aligned}
& f_{o, M}=\frac{1}{2 \pi\left(R_{\text {avg }} \pm \Delta R_{M}\right) C} \\
& Q=\frac{f_{o, M}}{B W}
\end{aligned}
$$

Yukarıda verilen eleman değerlerine göre tasarlanan 2. dereceden aktif yüksek geçiren filtre devresinde, giriş gerilimi $V_{\text {in }}(p-p)=1 \mathrm{~V}$ olan sabit sinüzoidal işaret için farklı frekanslarda ölçülen çıkış gerilimi ve hesaplanan kazanç, normalize kazanç, normalize kazanç desibel $(\mathrm{dB})$ değerleri Tablo 1 'de verilmiştir. Memristörlü 2. dereceden aktif yüksek geçiren filtre devresinde frekans1 arttırdıkça kazancımızın sabit kaldığı orta kazanç bandının olduğu yerde gerilim kazancı $A_{V \text { mid }_{M-C}}=5,88$ olduğu görülmüştür. $\mathrm{Bu}$ değeri daha önceden elde edilen kazanca oranlayarak normalize kazanç elde edilmiştir. Aynı işlemler direnç ile tasarlanan 2. dereceden aktif yüksek geçiren filtre devresinde de ele aldığımızda bu değeri $A_{V \text { mid }_{R-C}}=2,18$ olduğu görülmüştür. Elde edilen normalize kazanç değerlerini Bode grafiğgine aktarmak için bu değerlerin desibel noktaları Tablo 1'de hesaplanarak verilmiştir.

Tablo 1. 2. Dereceden aktif yüksek geçiren filtre devresinde farklı frekanslar için hesaplanan kazanç, normalize kazanç desibel $(\mathrm{dB})$ Değerleri $\left(V_{\text {in }}(p-p)=1 V, A_{V_{\text {mid }_{M-C}}}=5,88 A_{V_{\text {mid }_{R-C}}}=2,18\right)$

\begin{tabular}{ccccc}
\hline $\boldsymbol{F}(\boldsymbol{H z})$ & $V_{\text {out }}(\mathrm{p}-\mathrm{p})$ & $A_{V}=\frac{V_{\text {out }}}{V_{\text {in }}}$ & $\frac{A_{V}}{A_{V_{\text {mid }}}}$ & $20 \log \frac{A_{V}}{A_{V_{\text {mid }}}} d B$ \\
& $\boldsymbol{M}-\boldsymbol{C} / \boldsymbol{R}-\boldsymbol{C}$ & $\boldsymbol{M}-\boldsymbol{C} / \boldsymbol{R}-\boldsymbol{C}$ & $\boldsymbol{M}-\boldsymbol{C} / \boldsymbol{R}-\boldsymbol{C}$ & $\boldsymbol{M}-\boldsymbol{C} / \boldsymbol{R}-\boldsymbol{C}$ \\
\hline $0.5 \mathrm{~Hz}$ & $0.21 \mathrm{~V} / 0.26 \mathrm{~V}$ & $0,212 / 0,260$ & $0,036 / 0,119$ & $-28,86 /-8,46$ \\
$1 \mathrm{~Hz}$ & $676 \mathrm{mV} / 0.4 \mathrm{~V}$ & $0,676 / 0,40$ & $0,114 / 0,183$ & $-18,78 /-4,72$ \\
$1.5 \mathrm{~Hz}$ & $1,3 \mathrm{~V} / 540 \mathrm{mV}$ & $1,3 / 0,540$ & $0,221 / 0,247$ & $-13,10 /-2,12$ \\
$2 \mathrm{~Hz}$ & $1,8 \mathrm{~V} / 700 \mathrm{mV}$ & $1,8 / 0,700$ & $0,306 / 0,321$ & $-10,28 /-9,86$ \\
$3 \mathrm{~Hz}$ & $2,8 \mathrm{~V} / 960 \mathrm{mV}$ & $2,8 / 0,960$ & $0,476 / 0,44$ & $-6,44 /-7,12$ \\
$4 \mathrm{~Hz}$ & $3,58 \mathrm{~V} / 1 \mathrm{~V}$ & $3,58 / 1,2$ & $0,608 / 0,55$ & $-4,3 /-5,18$ \\
$5 \mathrm{~Hz}$ & $4,28 \mathrm{~V} / 1,34 \mathrm{~V}$ & $4,28 / 1,34$ & $0,727 / 0,614$ & $-2,75 /-4,22$ \\
$10 \mathrm{~Hz}$ & $5,68 \mathrm{~V} / 1,8 \mathrm{~V}$ & $5,68 / 1,8$ & $0,965 / 0,829$ & $-0,3 /-1,66$ \\
$\ldots$ & $\ldots$ & $\ldots$ & $\ldots$ & $\ldots$ \\
$100 \mathrm{~Hz}$ & $5,88 \mathrm{~V} / 2,18 \mathrm{~V}$ & $5,88 / 2,18$ & $1 / 1$ & $0 / 0$ \\
$1000 \mathrm{~Hz}$ & $5,88 \mathrm{~V} / 2,18 \mathrm{~V}$ & $5,88 / 2,18$ & $1 / 1$ & $0 / 0$ \\
\hline
\end{tabular}

Tablo 1'deki frekans ve normalize kazanç desibel $(\mathrm{dB})$ değerleri kullanılarak filtreye ait Bode eğrisi Şekil 12' de verilmiştir.

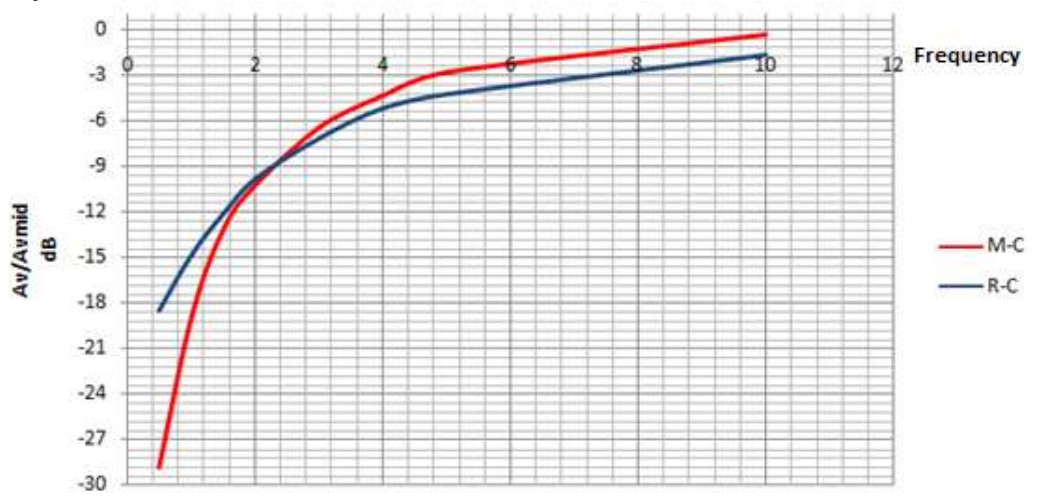

Şekil 12. 2. dereceden aktif yüksek geçiren $M-C$ ve R-C filtre devrelerinin bode grafiği

Şekil 12'de de gösterildiği gibi devremizin kesim frekansı yaklaşık olarak 5Hz'e ayarlanmıştır. Filtre devrelerinde duvar etkisi (wall effect) dediğimiz olayda eğimin dik olması istenir. Kesim frekansı dik eksene ne kadar yakın olursa o derece kaliteli bir süzgeç işlemi yapıldığı anlamına gelmektedir. 0-2 
$\mathrm{Hz}$ frekans aralığında $\mathrm{M}-\mathrm{C}$ ve $\mathrm{R}-\mathrm{C}$ devrelerinin kalite faktörleri incelendiğinde, $\mathrm{M}-\mathrm{C}$ filtre devresinin eğiminin yaklaşı 3 kat daha yüksek olduğu görülmüş̧ür. 2-5 Hz frekans aralığında, M-C devresinin kalite faktörünün R-C den daha iyi olduğu görülmüştür. 5-10 Hz frekans aralığında, M-C devresinin kalite faktörünün R-C'ye göre daha doğrusal bir eğri oluşturduğu izlenmiştir. Daha da yüksek frekanslarda memristör taklit devresi 2. Bölümde de belirttiğimiz gibi lineer direnç gibi davranmakta ve 2. Dereceden aktif YGF devresinde de $A v / A v_{-}$mid $=1$ olduğu hesaplanmıştır.

\section{Sonuç ve Öneriler}

$\mathrm{Bu}$ çalışmada öncelikle Mutlu ve arkadaşları tarafından geliştirilen lineer sürüklenme hızlı $\mathrm{TiO}_{2}$ memristör modeli, Alharbi ve arkadaşları tarafından önerilen gerilim kontrollü memristör ve Muthuswamy tarafından geliştirilen bir başka gerilim kontrollü memristör devreleri ele alınmıştır. Ele alınan taklit devrelerinin farklı frekans tepkileri altındaki akım-gerilim karakteristikleri incelenmiştir. Mutlu ve arkadaşları tarafindan geliştirilen gerilim kontrollü modelin uygulama deneylerinde en kararlı çıkış tepkisini veren, $150 \mathrm{~Hz}$ frekansta histerezis alanın en az olduğu yani tamamen lineer özellik gösteren ve daha az aktif devre elemanı ile oluşturulduğu görülmüş̧ür. Kullanılan bu model 2. dereceden aktif yüksek geçiren filtreye uyarlanarak kalite faktörü, akım-gerilim karakteristikleri, histerezis bölgeleri ve kesim frekansları incelenmiştir. Memristörlü 2. dereceden aktif YGF devresinde kesim frekansı yaklaşık olarak $5 \mathrm{~Hz}$ 'e ayarlanmıştır. M-C ve R-C devreleri $0-10 \mathrm{~Hz}$ frekans aralığında kalite faktörleri karşılaştırıldığında, M-C devresinin R-C devresine göre daha iyi sonuç verdiği görülmüştür. $0-2 \mathrm{~Hz}$ frekans aralığında eğimin (kesim frekansının band genişliğine olan oranı) R-C filtreden 3 katına kadar daha yüksek çıktığı, 2-5 Hz frekans aralığında, kalite faktörünün R-C den daha iyi olduğu, 5-10 $\mathrm{Hz}$ frekans aralığında, yani kesim frekansının üzerine çıktığımızda $\mathrm{M}-\mathrm{C}$ devresinin kalite faktörünün RC'ye göre daha doğrusal bir eğri oluşturduğu izlenmiştir. Bu modelin istenen amaç ve kullanımına göre devre elemanlarının değerleri değiştirilerek farklı kesim frekanslarında ve aktif filtrelerde, özellikle düşük frekanslarda çalış1lacak 2. Dereceden aktif YGF devrelerinde tercih edilebilir.

\section{Teşekkür}

Çalışma kapsamındaki uygulama ve hesaplamalar Van Yüzüncü Y11 Üniversitesi Elektronik Laboratuvarında yapılmıştır.

\section{Yazarların Katkısı}

Çalışmada tüm yazarlar eşit oranda katkı sunmuştur.

\section{Çıkar Çatışması Beyanı}

Yazarlar arasında herhangi bir çıkar çatışması bulunmamaktadır.

\section{Araştırma ve Yayın Etiği Beyanı}

Yapılan çalışmada, araştırma ve yayın etiğine uyulmuştur.

\section{Kaynaklar}

[1] Alharbi A.G., Fouda M.E., Chowdhury M.H. 2015. Memristor Emulator Based on Practical Current Controlled Model. IEEE 58th International Midwest Symposium on Circuits and Systems (MWSCAS), 1-4.

[2] Alharbi A.G., Fouda M.E., Khalifa Z.J., Chowdhury M.H. 2016. Simple Generic Memristor Emulator for Voltage-Controlled Models. In Circuits and Systems (MWSCAS), IEEE 59th International Midwest Symposium, 1-4.

[3] Biolek Z., Biolek D., Biolkova V. 2009. SPICE Model of Memristor with Nonlinear Dopant Drift. Radioengineering, 18 (2). 
[4] Chua L. 1971. Memristor-the Missing Circuit Element. IEEE Transactions on Circuit Theory, 18 (5): 507-519.

[5] Strukov D.B., Snider G.S., Stewart D.R., Williams R.S. 2008. The missing memristor found. Nature, 453: 80-83.

[6] Kirilov S. M., Yordanov R. S., Mladenov V. M. 2013. Analysis and Synthesis of Band-Pass and Notch Memristor Filters, In 17th WSEAS International Conference on Circuits.

[7] Megep. 2012. Oscillators and http://megep.meb.gov.tr/mte_program_modul/moduller_pdf/Osilat\%C3\%B6rler\%20Ve\%20Filt re\%20Devreleri.pdf. (Erişim Tarihi:12.07.2017).

[8] Muthuswamy B. 2010. Implementing Memristor Based Chaotic Circuits. International Journal of Bifurcation and Chaos, 20 (5): 1335-1350.

[9] Mutlu R., Karakulak E. 2009. Department of Electronics, A Memristor (Memory Resistor) Emulator Circuit for Using at Engineering Education. 13. Elektrik, Elektronik, Bilgisayar ve Biyomedikal Mühendisliği Ulusal Kongresi, 23-26 Aralık, ODTÜ, Ankara.

[10] Mutlu R., Karakulak E. 2010. Emulator Circuit of $\mathrm{TiO}_{2}$ Memristor with Linear Support Drift Made Using Analog Multiplier. In Electrical Electronics and Computer Engineering (ELECO), 380384.

[11] Sözen H., Çam U. 2013. On The Realization of Memristor Based RC High Pass Filter. 8th International Conference on Electrical and Electronics Engineering (ELECO), IEEE, 45-48.

[12] Talukdar A., Radwan A.G., Salama K.N. 2010. Time Domain Oscillating Poles: Stability Redefined in Memristor Based Wien-Oscillators. In 2010 International Conference on Microelectronics, IEEE, 288-291.

[13] Yener Ş.Ç., Mutlu R., Kuntman H.H. 2014. Performance Analysis of a Memristor-Based Biquad Filter Using a Dynamic Model. Informacije Midem-Journal of Microelectronics Electronic Components And Materials, 44 (2): 109-118.

[14] Williams R.S. 2008. How We Found the Missing Memristor. IEEE Spectrum, 45 (12). 